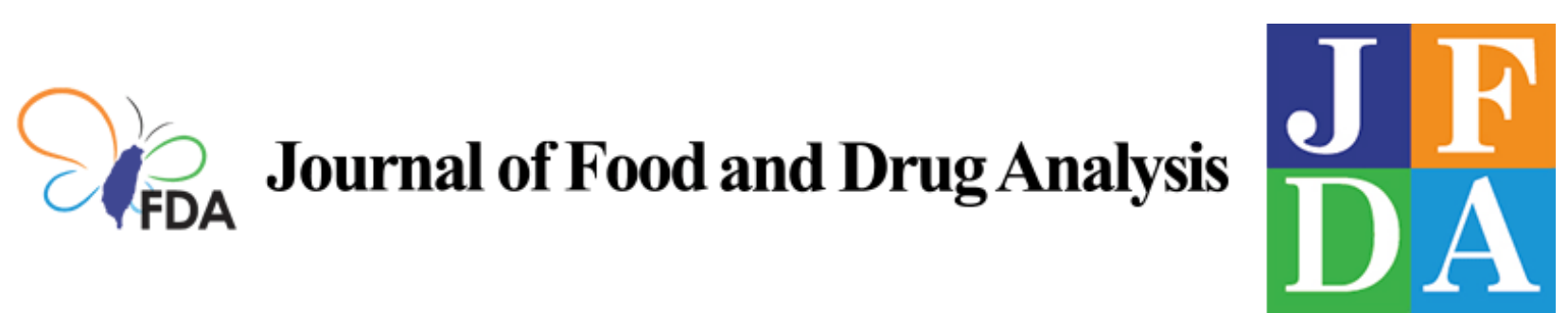

Volume 29 | Issue 1

Article 3

2021

\title{
N-nitrosodimethylamine (NDMA) Contamination of Ranitidine Products: A review of recent findings
}

Follow this and additional works at: https://www.jfda-online.com/journal

Part of the Medicinal and Pharmaceutical Chemistry Commons, Other Chemicals and Drugs Commons, Other Pharmacology, Toxicology and Environmental Health Commons, Other Pharmacy and Pharmaceutical Sciences Commons, and the Pharmaceutical Preparations Commons (c) (1) (9)

This work is licensed under a Creative Commons Attribution-Noncommercial-No Derivative Works 4.0 License.

\section{Recommended Citation}

Aldawsari, Fahad S.; Alshehri, Yahya M.; and Alghamdi, Thamer S. (2021) "N-nitrosodimethylamine (NDMA) Contamination of Ranitidine Products: A review of recent findings," Journal of Food and Drug Analysis: Vol. 29 : Iss. 1 , Article 3.

Available at: https://doi.org/10.38212/2224-6614.1133

This Review Article is brought to you for free and open access by Journal of Food and Drug Analysis. It has been accepted for inclusion in Journal of Food and Drug Analysis by an authorized editor of Journal of Food and Drug Analysis. 
N-nitrosodimethylamine (NDMA) Contamination of Ranitidine Products: A review of recent findings

Cover Page Footnote

Not Applicable 


\title{
$\mathrm{N}$-nitrosodimethylamine (NDMA) contamination of ranitidine products: $A$ review of recent findings
}

\author{
Fahad S. Aldawsari*, Yahya M. Alshehry, Thamer S. Alghamdi
}

Saudi Food and Drug Authority (SFDA), 12843-7116 Riyadh, Saudi Arabia

\begin{abstract}
Ranitidine is a medication that has been used to alleviate heartburn and other disorders for over 40 years. Following reports of $\mathrm{N}$-nitrosodimethylamine (NDMA) contamination in ranitidine products, there have been many recalls and registration suspensions. Here, we revise the literature information confirming ranitidine association with NDMA. Then, we highlight the documented mechanisms for NDMA release from ranitidine. In addition, the stability issue for this medicine is discussed. After that, we review and discuss the results of the United States Food and Drug Administration and the Australian Therapeutic Goods Administration laboratory testing of ranitidine products and the detected NDMA levels. Finally, the case of NDMA generation in Angiotensin II Receptor Blockers (ARBs) and ranitidine were compared in an attempt to address the circumstances leading to the current contamination.
\end{abstract}

Keywords: NDMA, Ranitidine, US FDA, Drug contamination, LC-MS

\section{Introduction}

$\mathrm{R}$ anitidine is a chemical compound that is available as over the counter (OTC) medicine to alleviate heartburn effects and gastric ulcers. During the Eighties, the sales of Zantac ${ }^{\circledR}$, the wellknown brand of ranitidine, has exceeded $\$ 1$ billion and Zantac ${ }^{\circledR}$ was then described as "the singlelargest-selling prescription drug, ever" [1]. Ranitidine belongs to a class of therapeutics known as $\mathrm{H}_{2}$ blockers, which also includes cimetidine, famotidine and nizatidine. The ranitidine chemical structure includes functional groups of nitro, secondary, and tertiary amines (Fig. 1).

$\mathrm{N}$-nitrosodimethylamine (NDMA) belongs to the nitrosamines group with the formula $\mathrm{C}_{2} \mathrm{H}_{6} \mathrm{~N}_{2} \mathrm{O}$, and has recently become the focus of the scientific community because of its unexpected occurrence among pharmaceutical impurities in some medications [2]. NDMA is recognized as a probable carcinogen by the International Agency for Research on Cancer (IARC) [2].
As of early September 2019, regulatory authorities have announced that ranitidine-containing medications are contaminated with unacceptable levels of NDMA impurities. Countries such as the USA [3], Canada [4], Singapore [5], Australia [6] and Switzerland [7] have recalled and/or suspended ranitidine containing products. Ranitidine is the second medication to be associated with NDMA impurities after the Angiotensin II Receptor Blockers (ARBs) medications, commonly known as "sartans", that were in 2018, announced to be contaminated with NDMA, as well as other nitrosamine compounds [8].

According to the literature, there are at least 20 studies in the last decade that repeatedly confirmed ranitidine as an NDMA precursor, particularly during water disinfection process [9-14]. Moreover, other studies assessed the quality attributes for this molecule and revealed some potential deficiencies. Despite this evidence, it seems that the issue of NDMA-release from ranitidine was, until recently, largely ignored by the pharmaceutical community.

The US Food and Drug Administration (FDA) [3] and the Australian Therapeutic Goods

Received 20 May 2020; revised 16 September 2020; accepted 12 October 2020.

Available online 15 March 2021.

* Corresponding author. Saudi Food and Drug Authority (SFDA), 12843-7116 Riyadh, Saudi Arabia.

E-mail address: fahad386@gmail.com (F.S. Aldawsari). 
<smiles>CNC(=NC#N)NCCSCc1nc[nH]c1C</smiles>
Cimetidine<smiles>NC(N)=Nc1nc(CSCC/C(N)=N/S(N)(=O)=O)cs1</smiles>

Famotidine<smiles>CN/C(=C\[N+](=O)[O-])NCCSCc1ccc(CN(C)C)o1</smiles>

Ranitidine<smiles>CNC(=C[N+](=O)[O-])NCCSCc1csc(CN(C)C)n1</smiles>

Nizatidine

Fig. 1. The chemical structure for ranitidine and the other $\mathrm{H}_{2}$ blockers.

Administration (TGA) [15] have released the results for NDMA in different ranitidine dosage forms which showed broad-range concentrations for this contaminant. Moreover, Different formulations mean different ingredients, which make it ambiguous to speculate the origin of NDMA contamination.

In this short review, we revisited potential mechanisms for NDMA release from ranitidine that originally reported in water research studies. In addition, we highlighted literature studies that evaluated the stability of ranitidine in liquid and solid forms. Moreover, we analyzed and discussed the results published by regulatory authorities, which included the detected NDMA levels in ranitidine and the corresponding dosage form. Finally, the case of NDMA in ARBs is compared with that of ranitidine, in an attempt to understand and identify potential sources in order to mitigate this contamination.

\section{Potential mechanisms for ranitidine degradation to NDMA}

In the literature, there are multiple reports concerning ranitidine degradation into NDMA. The chemical structure for ranitidine contains a lateral tertiary amine that is relevant to nitrosamine formation (Fig. 1). During the Seventies and Eighties, there were studies describing mechanisms for nitrosamine formation among molecules possessing tertiary amines. According to Loeppky et al. [16], nitrosamine generation necessitates three elements; nitrosatable substrate, nitrosating agent and specified reaction conditions. The tertiary amine functional group in ranitidine is considered the nitrosatable substrate by Loeppky definition [16].
Examples for nitrosating agents are nitric acid and nitrite [16]. Experimentally, chemical reactions revealed that nitrosamines generally can be formed at room temperatures (i.e. $25^{\circ} \mathrm{C}$ ), in addition to high temperatures such as $120^{\circ} \mathrm{C}$ [16-18]. These observations led to the suspicion that naturally occurring nitrite in the human body, might interact with potential nitrosatable drugs and generate nitrosamines $[19,20]$. During that era, extreme conditions (e.g. concentrated acids and high temperatures) were applied, in order to confirm and identify the nitrosamine formation. To that end, those earlier observations established the basics for the recent inadvertently findings of nitrosamines releasing medications.

During the last decade, ranitidine was frequently used as a NDMA precursor in chemical reactions occurred within water treatment processes (recognized as chloroamination) [13]. Generally, NDMA is believed to be produced during water treatment as a result from either classical nitrosation or through unsymmetrical dimethylhydrazine (UDMH) mechanism [12,13]. In one study, ranitidine presented a higher rate towards NDMA formation than other tested compounds [13]. Moreover, a different report discussed factors and methods to circumvent NDMA release form ranitidine, specifically during wastewater treatment [12]. In another study assessing the role of chloramine species in NDMA formation, ranitidine was found to form NDMA in the presence of either mono or dichloramine precursors, which suggest the high potential for ranitidine to generate NDMA under numerous amine precursors [14]. An in vitro kinetic study investigated the effects of nitrite, oxygen and $\mathrm{pH}$ on NDMA formation from ranitidine medicine [11]. This study proposed a complex 
mechanism where nitrite ion is anticipated to inhibit NDMA formation rate depending on the substrate concentration [11,12]. The above-cited reports provide insights into the complex mechanistic pathway for ranitidine-NDMA generation. Nonetheless, key elements in this reaction scheme seem to be crucial for NDMA reaction initiation, such as amine precursor, chloramine species, and well-controlled reaction conditions.

It seems that there is a borderline between the earlier nitrosamine studies and those documented in water treatment processes. In fact, the earlier studies have utilized rigorous as well as accelerated conditions, which might unlikely occur within ranitidine during the storage of the drug formulation. In contrast, the conditions used in water treatment studies were slow, and supported by kinetics, which might possibly explored to investigate the current situation in ranitidine. The next question is whether NDMA formation mechanisms reported in water treatment studies still be applicable for pharmaceutical dosage forms. Interestingly, there are two studies which may provide clues to this quest. Seid,
GM et al. [12] and Roux, LJ et al. [11] studied ranitidine degradation byproducts under simulated chloramination process using Liquid Chromatography Mass Spectrometry (LC-MS) and Gas Chromatography Mass Spectrometry (GC-MS) techniques. Based on the detected mass fragments, authors proposed mechanisms for NDMA generation from ranitidine (Fig. 2). Similarly, analysis of ranitidine fragments (using mass spectrometry) in the contaminated pharmaceutical samples might be utilized to correlate, if any, the observed mass fragments to those reported in water research. Additionally, this attempt may also reveal the identity for key molecules in order to postulate the overall mechanism for ranitidine degradation among pharmaceutical products.

\section{Stability of ranitidine}

Stability of ranitidine was assessed in previous studies, which enlighten the inherent stability issue for this medicine. For instance, a study that was published in the Nineties, revealed that the
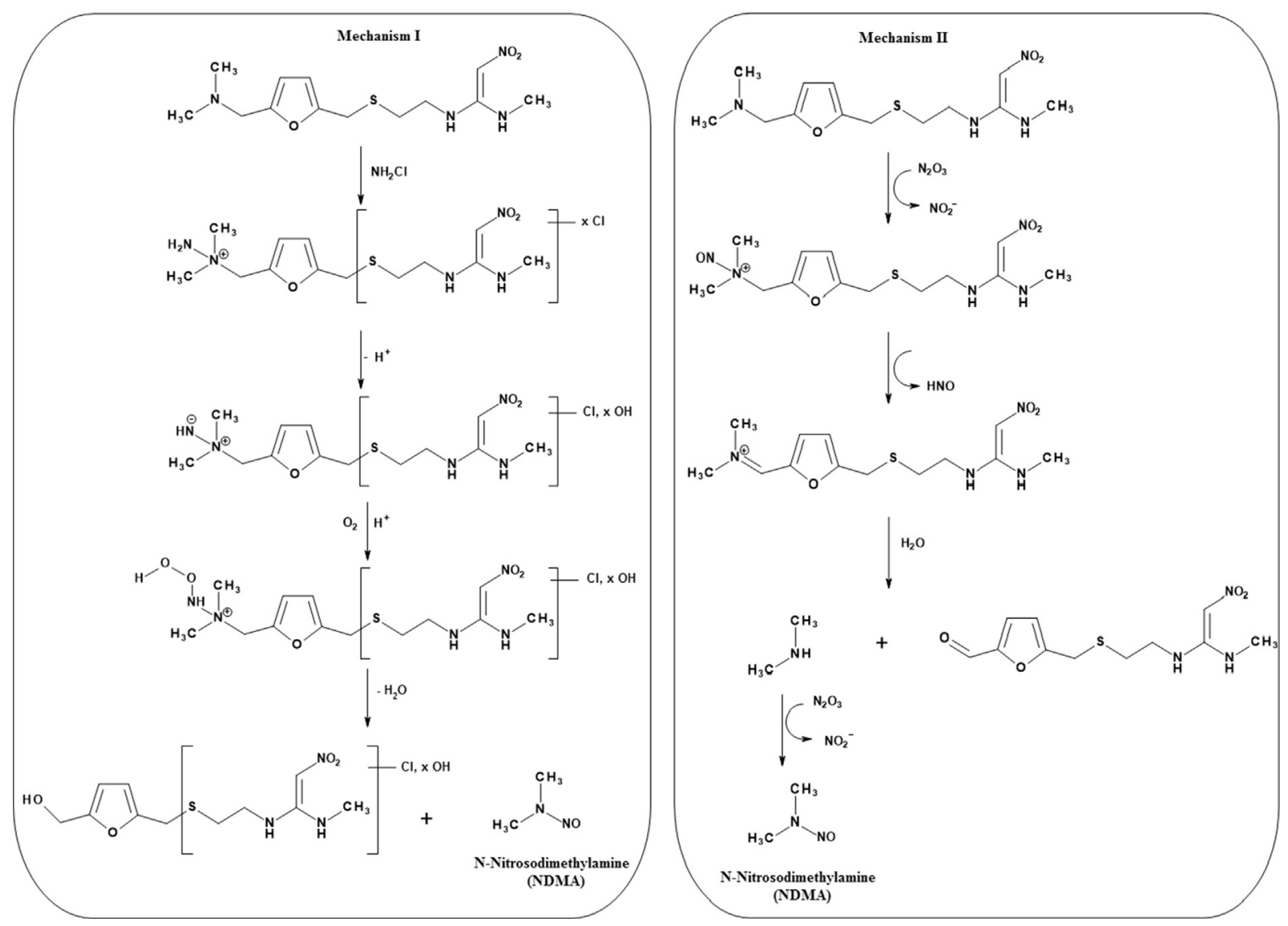

Fig. 2. Examples of proposed mechanisms for NDMA generation from ranitidine. Reprinted (adapted) with permission from Ref. [11]. Copyright (2012) American Chemical Society. 
temperature of $45{ }^{\circ} \mathrm{C}$ and absolute humidity of $45 \%$, caused ranitidine to degrade in about $20 \%$ [21]. Another study showed that ranitidine at $\mathrm{pH}$ of 5-8, had the slowest rate of degradation [22]. In a different study aimed at identification and quantification for degradation products of ranitidine, three molecules were observed, namely alcohol, oxime and amine [23]. Those degradants were found to be formed in a percentage of 9,32 and $28 \%$ respectively [23]. Lastly, one report assessed the stability of ranitidine in an injectable solutions [24]. It was shown that ranitidine was unstable at temperatures of 40 and $55{ }^{\circ} \mathrm{C}$, since ranitidine samples failed the stability testing [24].

There was an interesting study which found unusual degradation products of ranitidine as a result of photo-irradiation. Jamrogiewicza, M. and Wielgomasb, B. [25] exposed ranitidine in its solid state to a xenon lamp at $45{ }^{\circ} \mathrm{C}$, and they have detected sixteen degradation products. Authors were surprised to find $\mathrm{N}, \mathrm{N}$-dimethylformamide (DMF) among the degradation products. It was confirmed that DMF was only formed when the sample was irradiated, since the non-irradiated counterpart did not show DMF signal [25]. Another study tested the oxidative degradation of $\mathrm{H}_{2}$ blockers after their exposure to $10 \% \mathrm{H}_{2} \mathrm{O}_{2}$ for 15 min using HPTLC [26]. It was found that both ranitidine and nizatidine similarity decreased in their concentrations, but famotidine did not [26]. This finding illustrates the influence of chemical structure and its contributions to the degradation process. To conclude, the issue of ranitidine instability is evident though literature data and the recent NDMA findings in ranitidine might be a consequence of concealed quality attribute for this medication.

\section{NDMA laboratory results from competent authorities}

It seems that the only available laboratory results for NDMA traces in ranitidine samples are those published by the Australian TGA [15] and the United States FDA [3]. The laboratory analysis released by TGA provided much more information that those released by FDA. The TGA provided results for different dosage forms, which included tablets, ampoules, syrups and effervescent tablets, with the number of batches tested as 30 , two, one, and one respectively. Despite the vast different in the number of samples tested for each dosage form, generally speaking, tablets demonstrated higher NDMA levels compared to liquids (ampoules and oral liquid). There was a general trend in tablets results for NDMA content: that products soon to be expired (manufactured earlier) contained higher NDMA levels that those manufactured recently (will expire later). That is clearly demonstrated by comparing NDMA levels (5.1 and $0.7 \mathrm{ppm}$ ) for batches with an expiry date of June 2019 and February 2022, respectively. These findings are in agreement with a previous study, which demonstrated experimentally that NDMA formation from ranitidine could take up to five days to reach measurable levels [9]. Contrary to tablets, for oral liquid, the batch with later expiry had more NDMA compared to the batch expiring sooner. Nevertheless, this cannot be conclusive, as there was only one batch that was tested.

According to the TGA results, the batches contained $<0.1$ ppm NDMA had an expiry date of October 2020 or beyond, which might points out to that, at the time of testing, the NDMA traces were very low (below instrumental detection limits), but might accumulate over time. This observation supports the speculation that NDMA is formed during the storage of ranitidine product and is not related to the manufacturing process.

For the majority of batches tested (by different manufactures), it can be observed that NDMA was detectable in the vast majority of tested batches. This is further supported by FDA results, which stated that NDMA was present in all tested batches. This might indicate that the problem is attributed to the Active Pharmaceutical Ingredient (API) itself and the associated storage conditions.

The FDA laboratory results included 12 tablets, two syrups, and four nizatidine products with multiple batches. The maximum NDMA levels observed in syrups were $1.37 \mathrm{ppm}$, compared to $2.85 \mathrm{ppm}$ for tablets. In addition to ranitidine, the FDA has tested another $\mathrm{H}_{2}$ blocker, nizatidine, which is chemically similar to ranitidine. The average NDMA levels in nizatidine were lower than that of ranitidine. This finding is supported by two previous studies. The first was a computational study [10] that demonstrated that ranitidine had, by far, a high potential to release NDMA under chloramination processes compared to nizatidine, with activation free energies of 12.6 and $21.1 \mathrm{kcal} / \mathrm{mol}$ for ranitidine and nizatidine, respectively. The second is a practical study [9] that reported that ranitidine had a 2-fold higher capability for producing NDMA compared to nizatidine under the tested conditions.

The diverse NDMA concentrations among the different formulations might suggest that the 
NDMA formation mechanism is different. Considering that injectables, effervescent tables, film coated tables and syrups are diversely manufactured, determination of NDMA mechanisms might be complex. Furthermore, other factors involved in nitrosamine formation such as $\mathrm{pH}$, oxygen, temperature and substrate concentration increase the complexity for such determination. Deep investigation by the analysis of every ingredient in each formulation, packaging, inking, or even the water used during manufacturing need to be performed. There was an uncertain report which claimed that packaging material (nitrocellulose) might be a contributing factor in NDMA formation process [27]. However, it appears that no confirmation data is available in this regards.

\section{Ranitidine versus sartan}

The case of ranitidine seems to be different from that of sartan. According to the scientific report by the European Medicines Agency (EMA) [28], NDMA was an impurity that was carried through manufacturing until the finished product. NDMA was formed during the synthesis of certain chemical moiety (tetrazole) [28] among the chemical structure of ARBs drug. Not all ARB products showed positive NDMA. Hence, the contamination issue occurred only at certain manufacturing facilities (or a specific API source to be exact), which in part was attributed to using contaminated solvent [28] (Fig. 3).

The case of ranitidine so far exhibits a somewhat unknown reaction between the API itself and/or with other ingredients of the dosage form that lead to NDMA formation. The finding that NDMA is absent in the recently manufactured batches, and subsequently elevating upon time, is a clear discrepancy between NDMA occurrences in ranitidine compared to ARBs. Nevertheless, detecting DMF as a degradation product after exposing ranitidine to light is suspicious because DMF was involved in the NDMA contamination of ARBs (Fig. 3). Further research is needed to confirm whether DMF is present in the contaminated ranitidine products, and to correlate, if any, the output of this investigation to the NDMA formation mechanism.

\section{Possible sources of NDMA in ranitidine}

Taking the case for ranitidine from a broad prospective, NDMA traces could have an API origin, non-API origin, or a mixture of both. The API possibility is supported by three facts. The first is that the FDA has stated that NDMA was detected in all batches tested (tablets and liquids). The second is that TGA has tested additional dosage forms (ampoules and effervescent tablets, not covered by the US FDA), and NDMA was also found in those samples. The third is that ranitidine at accelerated conditions (represented by high temperatures) using the GC-MS instrument has released NDMA $[2,29,30]$. It should be noted that different dosage forms yielded NDMA traces, which might weaken the hypothesis that NDMA originates from other ingredients/packaging. Nevertheless, one report has suggested that blister packaging [27] might have a
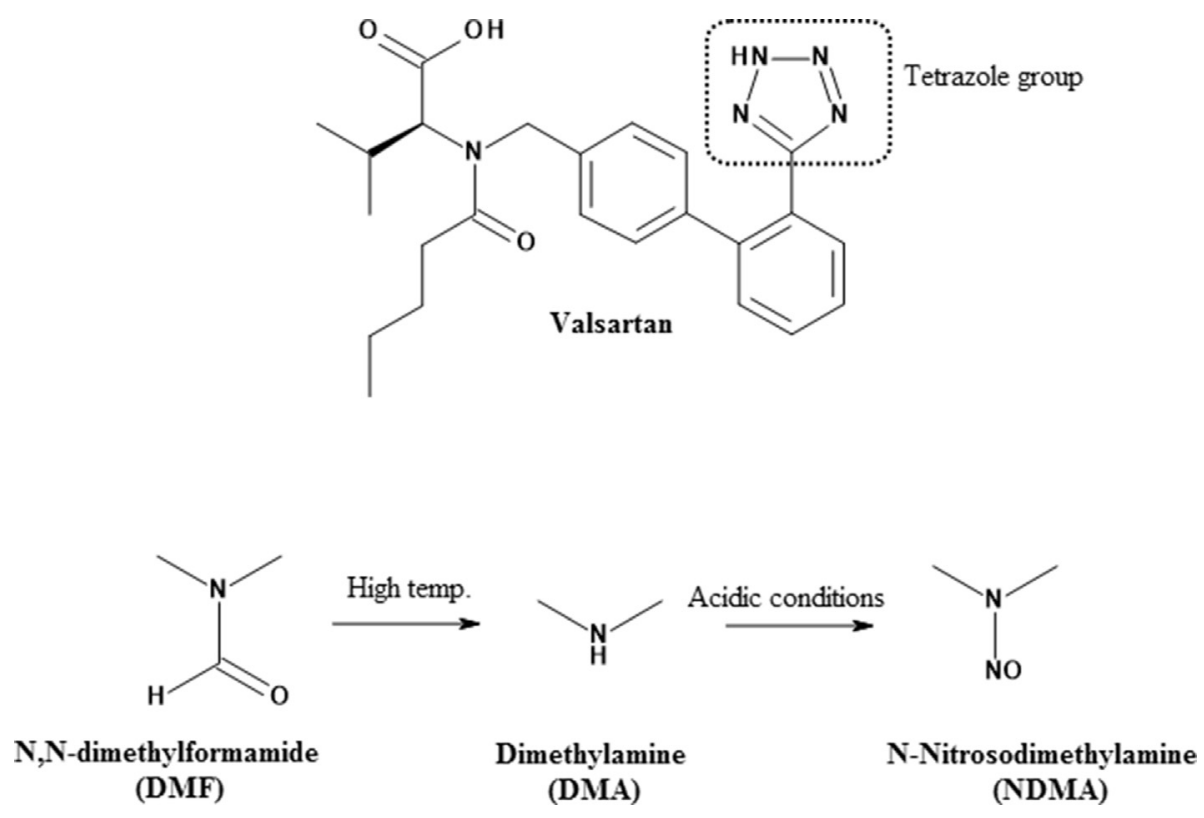

Fig. 3. Chemical structure for a selected sartan medication and a proposed mechanism for NDMA generation among these therapeutics. 
role in this process, as some ingredients (nitrocellulose) might be able to react with printing ink (amines in nature) and could ultimately produce NDMA in the final product. Of note, the last thought is not applicable to liquids. Consequently, NDMA impurities in ranitidine products might be a result of both (API and non-API) origins.

Recently, a study by Yasuhiro ABE et al. [30] investigated the NDMA formation in ranitidine solid forms that were represented by tablets and reagent powders. To simulate storage conditions, the authors tested the influence of factors such as temperature, oxygen and nitrite on the quantified NDMA levels measured by LC-MS. In agreement with the US FDA and TGA, NDMA levels in ranitidine showed a proportional increase within time at the tested temperatures $\left(40\right.$ and $\left.50{ }^{\circ} \mathrm{C}\right)$. Moreover, it was demonstrated that in the absence of oxygen, NDMA levels were minimized. Interestingly, the quantified NDMA levels in ranitidine reagent powders were by far lower than those detected in tablets, which point out to the possible impact of tableting process on NDMA generation. Furthermore, nitrite was shown to be accumulating during the storage of ranitidine samples, however, no clear relationship was observed [30]. It is noteworthy that factors such as temperature, oxygen and nitrite were initially involved in the NDMA generation during the water treatment process. The new findings from ranitidine medications reinforce the importance of the previous water treatment studies and provide a chance to re-investigate other factors in order to identify the possible mechanisms for NDMA formation in ranitidine products.

Manufactures or researchers investigating NDMA case in ranitidine might consider factors or circumstances that mitigate or potentially prevent NDMA formation in ranitidine. Literature data suggests two strategies which may possibly interfere with NDMA generation within ranitidine molecule. Firstly, a kinetic study demonstrated that nitrite ion is capable of inhibiting an intermediate formation among the proposed mechanism, ultimately leading to less NDMA production [12]. Secondly, an elegant study revealed that ranitidine complexation with $\beta$ cyclodextrin was effective in reducing volatile photodegradation products, including the DMF [31]. Additional research is needed to initially elucidate the NDMA generation mechanisms in ranitidine pharmaceutical products, and then possible mitigation strategies might be explored.

During the preparation of this manuscript, the US FDA has released a public statement asking the manufactures to withdraw all the batches and formulations of ranitidine. It also advised all healthcare professionals to cease the prescription of this medicine. This decision came after the laboratory findings of unacceptable levels of NDMA formed under uncontrolled and controlled storage conditions. The agency did not comment on the fate of nizatidine, as this ranitidine alternative previously showed less NDMA than ranitidine. Nizatidine might still be under investigation. Other international regulatory agencies have not commented on this action taken by FDA. The factors and circumstances discussed here in this short review could give ranitidine a second chance following a re-evaluation of its manufacturing processes. Developing novel strategies to mitigate or prevent NDMA formation could be potential option to provide a new horizon for the continued use of this effective medication.

\section{Conclusion}

Ranitidine is a clinically useful medication that is used to control many disorders. The discovery that ranitidine generates NDMA is likely associated with its intrinsic structural features and/or incompatibility with the inactive ingredients among the pharmaceutical formulations. The mechanism of NDMA production in ranitidine products need to be determined and a tool for that would be analyzing the mass fragments in the defected products. Future strategies to mitigate NDMA production in ranitidine could be taken in consideration in order to save this clinically effective medicine.

\section{Conflict of interest}

None.

\section{References}

[1] Companies search for next $\$ 1$ billion drug. The New York Times; 1988. Available at: https://www.nytimes.com/1988/11/ 28/business/companies-search-for-next-1-billion-drug.html. [Accessed 2 September 2020].

[2] White CM. Understanding and preventing (N-nitrosodimethylamine) NDMA contamination of medications. Ann Pharmacother 2020;54:611-4.

[3] FDA requests remoal of all ranitidine products (zantac) from the market. U.S. Food and drug administration. Available at: https://www.fda.gov/news-events/press-announcements/ fda-requests-removal-all-ranitidine-products-zantac-market. [Accessed 29 March 2020].

[4] Health Products Info Watch, January 2020. Health Canada. Available at: https://www.canada.ca/en/health-canada/ services/drugs-health-products/medeffect-canada/healthproduct-infowatch/january-2020.html. [Accessed 2 March 2020].

[5] HSA stops supply of eight brands of ranitidine products in Singapore. Health Sciences Authority; 2019. Available at: https://www.hsa.gov.sg/announcements/news/hsa-stopssupply-of-eight-brands-of-ranitidine-products-in-singapore. [Accessed 22 November 2019].

[6] TGA investigation - potential contamination with N-nitrosodimethylamine. Therapeutic Goods Administration. 
Available at: https://www.tga.gov.au/alert/ranitidine. [Accessed 20 March 2020].

[7] Recall of all preparations containing ranitidine from the Swiss market. Swissmedic; 2019. Available at: https://www.swissme dic.ch/swissmedic/en/home/news/mitteilungen/rueckrufe praeparaten_ranitidin.html. [Accessed 5 December 2019].

[8] Masada S, Tsuji G, Arai R, Uchiyama N, Demizu Y, Tsutsumi $\mathrm{T}$, et al. Rapid and efficient high-performance liquid chromatography analysis of $\mathrm{N}$-nitrosodimethylamine impurity in valsartan drug substance and its products. Sci Rep 2019;9:11852.

[9] Le Roux J, Gallard H, Croue JP. Chloramination of nitrogenous contaminants (pharmaceuticals and pesticides): NDMA and halogenated DBPs formation. Water Res 2011;45: 3164-74.

[10] Liu YD, Selbes M, Zeng C, Zhong R, Karanfil T. Formation mechanism of NDMA from ranitidine, trimethylamine, and other tertiary amines during chloramination: a computational study. Environ Sci Technol 2014;48:8653-63.

[11] Roux JL, Gallard H, Croue JP, Papot S, Deborde M. NDMA formation by chloramination of ranitidine: kinetics and mechanism. Environ Sci Technol 2012;46:11095-103.

[12] Seid MG, Cho K, Lee C, Park HM, Hong SW. Nitrite ion mitigates the formation of N-nitrosodimethylamine (NDMA) during chloramination of ranitidine. Sci Total Environ 2018; 633:352-9.

[13] Shen R, Andrews SA. Demonstration of 20 pharmaceuticals and personal care products (PPCPs) as nitrosamine precursors during chloramine disinfection. Water Res 2011;45: 944-52.

[14] Selbes M, Beita-Sandi W, Kim D, Karanfil T. The role of chloramine species in NDMA formation. Water Res 2018;140: $100-9$.

[15] TGA Laboratories testing of ranitidine medicines. Therapeutic Goods Administration. 2019. Available at: https://www.tga.gov. au/tga-laboratories-testing-ranitidine-medicines. [Accessed 19 February 2020].

[16] Loeppky RN, Bao YT, Bae J, Yu L, Shevlin G. Blocking nitrosamine formation. Nitrosamines and related $N$-nitroso compounds. ACS symposium series., vol. 553. American Chemical Society; 1994. p. 52-65.

[17] Loeppky RN, Outram JR, Tomasik W, Faulconer JM. Rapid nitrosamine formation from a tertiary amine: the nitrosation of 2-(N,N-Dimethylaminomethyl)pyrrole. Tetrahedron Lett 1983;24:4271-4.

[18] Loeppky RNTW, Millard TG. Ester-mediated nitrosamine formation from nitrite and secondary or tertiary amines. In: O'Neill IK vBR, Miller CT, Long J, Bartsch H, editors. Nnitroso compounds: occurrence, biological effects and relevance to human cancer, s. 57. IARC Scientific Publications; 1984. p. 353-63.
[19] Rao G. N-nitrosamines from drug and nitrite: potential source of chemical carcinogens in humans? Pharm Int 1980;1: 187-90.

[20] Castegnaro M, Pignatelli B, Walker EA. Analysis of volatile $\mathrm{N}$-nitrosamines in commercial drugs. Food Chem Toxicol 1981;19:489-91.

[21] Teraoka R, Otsuka M, Matsuda Y. Effects of temperature and relative humidity on the solid-state chemical stability of ranitidine hydrochloride. J Pharmaceut Sci 1993;82:601-4.

[22] Haywood PA, Martin-Smith M, Cholerton TJ, Evans MB. Isolation and identification of the hydrolytic degradation products of ranitidine hydrochloride. Journal of the Chemical Society, Perkin Transactions 1987;1:951-4.

[23] Guerrieri PP, Smith DT, Taylor LS. Phase behavior of ranitidine $\mathrm{HCl}$ in the presence of degradants and atmospheric moisture-impact on chemical stability. Langmuir 2008;24: 3850-6.

[24] Vehabovic M, Hadzovic S, Stambolic F, Hadzic A, Vranjes E, Haracic E. Stability of ranitidine in injectable solutions. Int J Pharm 2003;256:109-15.

[25] Jamrogiewicz M, Wielgomas B. Detection of some volatile degradation products released during photoexposition of ranitidine in a solid state. J Pharmaceut Biomed Anal 2013;76: 177-82.

[26] Ahmed S, Elshaboury SR, Mohamed NA, Farrag S. Development of a validated comparative stability-indicating assay method for some H2-receptor antagonists. J Chromatogr Sci 2017;55:818-31.

[27] Update. New EMA requirements on nitrosamines - what ALL pharma companies need to know. Pharmaceutical Online; 2020. Available at: https://www.pharmaceuticalonline. $\mathrm{com} / \mathrm{doc} / \mathrm{new}$-ema-requirements-on-nitrosamines-what-allpharma-companies-need-to-know-0001. [Accessed 25 March 2020].

[28] Nitrosamines impurities. European Medicines Agency; 2020. Available at: https://www.ema.europa.eu/en/documents/ variation-report/sartans-article-31-referral-chmpassessment-report_en.pd. [Accessed 13 January 2020].

[29] Alshehri YM, Alghamdi TS, Aldawsari FS. HS-SPMEGC-MS as an alternative method for NDMA analysis in ranitidine products. J Pharmaceut Biomed Anal 2020;191: 113582.

[30] Abe Y, Yamamoto E, Yoshida H, Usui A, Tomita N, Kanno H, et al. Temperature-dependent formation of $\mathrm{N}$-nitrosodimethylamine during the storage of ranitidine reagent powders and tablets. Chem Pharm Bull (Tokyo) 2020.

[31] Jamrogiewicz M, Wielgomas B, Strankowski M. Evaluation of the photoprotective effect of beta-cyclodextrin on the emission of volatile degradation products of ranitidine. J Pharmaceut Biomed Anal 2014;98:113-9. 